\title{
Can ferritin/lymphocyte percentage ratio, a new indicator, predict the clinical course of COVID-19 cases?
}

\author{
Aygun H, Eraybar S \\ University of Health Sciences Turkey, Bursa Yuksek Ihtisas Training and Research Hospital, \\ Emergency Department, Bursa Turkey. sunaeraybar@gmail.com
}

\section{ABSTRACT}

OBJECTIVES: COVID-19 maintains its seriousness as a global emergency with its rapid distribution worldwide. Ferritin/lymphocyte percentage ratio (FLPR) may appear as a prognostic value at the initial evaluation stage and thus can be used as a simple, effective and reliable parameter in identification of patients critically ill with COVID-19.

METHODS: In this retrospective cohort study we evaluated patients who were hospitalized after being diagnosed with COVID-19 based on positive PCR results. We calculated FLPRs and classified disease severity due to initial emergency evaluation. The relationship between the severity of the need for hospitalization and intensive care, and 28-day mortality with FLPR were evaluated.

RESULTS: The differences between the groups as for COVID-19 severity and FLPR means were statistically significant $(x 2=148.284 ; S D=3 ; p=0.000)$. FLPR levels were found to be high in groups with critically and seriously ill patients. In the ROC analysis for the FLPR level, the area under the curve (AUC) value was found to be 0.909 (95\% Cl 0.857-0.961). When the cut-off value of FLPR was 21.11, the sensitivity and specificity were $82.9 \%$ and $82.8 \%$, respectively.

CONCLUSION: FLPR, a new parameter, can be used as a significant marker to predict the 28-day mortality patients (Tab. 6, Fig. 1, Ref. 25). Text in PDF www.elis.sk

KEY WORDS: COVID-19, percentage of lymphocytes, ferritin, FLPR, disease severity, emergency department.

\section{Introduction}

In December 2019, an acute respiratory disease, now known to be caused by a novel coronavirus, emerged in Wuhan, Hubei district, China. The disease quickly spread around Wuhan $(1,2)$. On January 30, 2020, the World Health Organization recognized this rapidly spreading infectious disease as an international public health emergency, currently known as a coronavirus disease 2019 (COVID-19), and then defined it as a pandemic on March 11, 2020 (3).

COVID-19 disease is a systemic infection with a significant effect on the hematopoietic system. Its incubation period (approximately 1-14 days) is characterized by nonspecific symptoms and normal or slightly decreased peripheral leukocyte and lymphocyte levels. With the viremia developing in the following 7-14 days, SARS Cov-2 causes an increase in angiotensin-converting enzyme-2 (ACE-2) levels especially in the lungs, heart and gastrointestinal system and a systemic inflammatory response defined

University of Health Sciences Turkey, Bursa Yuksek Ihtisas Training and Research Hospital, Emergency Department, Bursa Turkey

Address for correspondence: S. Eraybar, Assoc Prof, Yuksek Ihtisas Training and Research Hospital, Emergency Department, Mimar Sinan Mah. Emniyet Cad. Polis Okulu Karsisi Yildirim, Bursa, Turkey. Phone: +905325782903 as a 'cytokine storm' (4). Lymphocytes having ACE receptors on their surfaces are directly lysed by this effect. Interleukins and tumor necrosis factor alpha released in cytokine storm trigger lymphocyte apoptosis and simultaneously impair lymphocyte turnover by affecting lymphoid organs $(5,6)$. Although its mechanism is not clearly defined, lymphopenia appears as a cardinal finding at this stage.

Biomarkers of inflammatory response have been frequently evaluated during the follow-up of this inflammatory response and used as a measure for predicting mortality. Although there is a significant correlation between the percentage of neutrophils and neutrophil count, the neutrophil count percentage (NCP) is often used in analytical control of infections. In cases such as community-acquired pneumonia where lymphocytes' response is observed in the foreground, some studies have shown that the percentage of lymphocytes is an important marker in predicting the mortality risk (7). In the presence of high levels of white blood cells, a low lymphocyte percentage has been found to be significantly correlated in terms of hospitalization and mortality. Therefore, lymphocyte percentage is associated with poor results $(8,9)$.

One of the poor prognostic markers in COVID-19 patients is the ferritin level. Ferritin H-chain inactivates the secretion of inflammatory cytokines by activating macrophages. It is the pathogenesis of hyperferritinemic syndrome that can be explained in COVID-19 infection (10). Wu et al. showed the relationship between 
high serum ferritin levels and development of acute respiratory distress syndrome (ARDS). In addition, there are some studies associated with mortality rates and ferritin levels $(11,12)$. As a result of this significant change in lymphocyte number and percentage, and ferritin level, the use of these values together in COVID-19 cases may present an opportunity to develop a different approach.

In emergency services where COVID-19 patients are primarily evaluated, it is important to identify the critical patients, es-

Tab. 1. Demographic characteristics of patients.

\begin{tabular}{|c|c|c|c|}
\hline & & Frequency & $\%$ \\
\hline \multirow{2}{*}{ Gender } & Female & 174 & 52.6 \\
\hline & Male & 157 & 47.4 \\
\hline \multirow{2}{*}{ Comorbidities } & Yes & 176 & 53.2 \\
\hline & No & 155 & 46.8 \\
\hline \multirow{4}{*}{ Severity } & Critical & 42 & 12.7 \\
\hline & Serious & 49 & 14.8 \\
\hline & Moderate & 131 & 39.6 \\
\hline & Mild & 109 & 32.9 \\
\hline \multirow{4}{*}{ Thorax CT } & Typical & 111 & 33.5 \\
\hline & İntermediate & 120 & 36.3 \\
\hline & Atypical & 43 & 13 \\
\hline & Negative & 57 & 17.2 \\
\hline \multirow{10}{*}{ Symptom } & None & 12 & 3.6 \\
\hline & Fever & 35 & 10.6 \\
\hline & Diarrhea & 41 & 12.4 \\
\hline & Cough & 69 & 20.8 \\
\hline & Dyspnea & 48 & 14.5 \\
\hline & Weakness-myalgia & 41 & 12.4 \\
\hline & Fever, cough & 50 & 15.1 \\
\hline & Fever + dyspnea & 13 & 3.9 \\
\hline & Fever + cough + dyspnea & 19 & 5.7 \\
\hline & Loss of consciousness & 3 & 0.9 \\
\hline \multirow{2}{*}{ 28-day mortality } & Yes & 41 & 12.4 \\
\hline & No & 290 & 87.6 \\
\hline Total & & 331 & 100 \\
\hline
\end{tabular}

pecially at the first stage, and to implement appropriate and rapid diagnosis and treatment procedures for their survival. Additionally, it is important to use first-line examinations such as hemogram and biochemistry tests and to predict the critical patients at this stage in terms of providing effective treatment. The proportional interpretation of ferritin and lymphocyte percentage values, which are included in routine examinations, appear to be of prognostic value already at the initial evaluation stage and thus can be used as a simple parameter that can be effectively used as a reliable measure for identifying critical patients.

This study aims to evaluate the relationship between ferritin/ lymphocyte percentage ratio (FLPR) calculated in patients with COVID-19 disease with the severity classification of the disease and to determine its prognostic significance in terms of mortality in the course of the disease.

\section{Materials and methods}

All patients over the age of 18 who were hospitalized for COVID-19 during April 2020, admitted to our emergency department and whose COVID-19 polymerase chain reaction (PCR) test results were positive were retrospectively evaluated.

In addition to the demographic findings such as age and gender, vital signs at the time of admission, oxygen or advanced airway support applications in the emergency room, laboratory tests results, CT findings, and 28-day living status were recorded using patient files stored in the hospital automation system. Patients' CT results were classified as being typical, intermediate, atypical or negative according to the Radiological Society of North America Expert Consensus Statement on Reporting Chest CT Findings (13). The typical results were peripheral, bilateral ground glass opacities (GGO) with or without consolidation or visible intralobular lines, multifocal GGO of rounded morphology or visible intralobular

Tab. 2. Patients' clinical and demographic characteristics as per severity classification.

\begin{tabular}{|c|c|c|c|c|c|c|c|c|}
\hline & & & \multicolumn{4}{|c|}{ Severity } & \multirow{2}{*}{ Total } & \\
\hline & & & Critical & Severe & Moderate & Mild & & \\
\hline \multirow{4}{*}{ Gender } & Femple & Count & 18 & 14 & 77 & 65 & 174 & \multirow{4}{*}{$\mathrm{X} 2=17.11, \mathrm{p}<0.05$} \\
\hline & Female & Within gender & $10.3 \%$ & $8.0 \%$ & $44.3 \%$ & $37.4 \%$ & $100.0 \%$ & \\
\hline & & Count & 24 & 35 & 54 & 44 & 157 & \\
\hline & Male & Within gender & $15.3 \%$ & $22.3 \%$ & $34.4 \%$ & $28.0 \%$ & $100.0 \%$ & \\
\hline \multirow{4}{*}{ Comorbidities } & & Count & 32 & 34 & 67 & 43 & 176 & \multirow{4}{*}{$\mathrm{X} 2=22.57, \mathrm{p}<0.001$} \\
\hline & Yes & Within comorbidities & $18.2 \%$ & $19.3 \%$ & $38.1 \%$ & $24.4 \%$ & $100.0 \%$ & \\
\hline & & Count & 10 & 15 & 64 & 66 & 155 & \\
\hline & No & Within comorbidities & $6.5 \%$ & $9.7 \%$ & $41.3 \%$ & $42.6 \%$ & $100.0 \%$ & \\
\hline \multirow{8}{*}{ Thorax CT } & & Count & 18 & 31 & 47 & 15 & 111 & \multirow{8}{*}{$\mathrm{X} 2=116.73, \mathrm{p}<0.00$} \\
\hline & Typical & On thorax CT & $16.2 \%$ & $27.9 \%$ & $42.3 \%$ & $13.5 \%$ & $100.0 \%$ & \\
\hline & & Count & 19 & 16 & 63 & 22 & 120 & \\
\hline & Intermediate & On thorax CT & $15.8 \%$ & $13.3 \%$ & $52.5 \%$ & $18.3 \%$ & $100.0 \%$ & \\
\hline & & Count & 4 & 1 & 12 & 26 & 43 & \\
\hline & Atypical & On thorax CT & $9.3 \%$ & $2.3 \%$ & $27.9 \%$ & $60.5 \%$ & $100.0 \%$ & \\
\hline & & Count & 1 & 1 & 9 & 46 & 57 & \\
\hline & Negative & On thorax CT & $1.8 \%$ & $1.8 \%$ & $15.8 \%$ & $80.7 \%$ & $100.0 \%$ & \\
\hline \multirow{2}{*}{ Total } & & Count & 42 & 49 & 131 & 109 & 331 & \\
\hline & & On thorax CT & $12.7 \%$ & $14.8 \%$ & $39.6 \%$ & $32.9 \%$ & $100.0 \%$ & \\
\hline
\end{tabular}

CT - Computed tomography 
lines, reverse halo sign or other findings of an organizing pneumonia.

Based on the initial assessment of vital signs and test results, and in line with the clinical severity classification of COVID-19, the patients were separated in groups, namely mild (presence of mild symptoms and radiological findings that were normal or not suggestive of pneumonia), moderate (respiratory complaints and fever, presence of pneumonia upon radiological examination), severe (dyspnea, respiratory rate $30 /$ min, blood oxygen saturation $\leq 93 \%, \mathrm{paO}_{2} /$ $\mathrm{FiO}_{2}<300$ and/or $>50 \%$ of lung area of infiltrates within 24-48 hours) and critical (respiratory failure and need for mechanical ventilation, septic shock and/or multiple organ failure, and need for intensive care follow-up and treatment) (14).

FLPR was calculated by recording the lymphocyte percentage values and ferritin level obtained with hemogram taken in the emergency room at the time of first admission. The relationship between FLPR, severity of patients' clinical status, and 28-day mortality was evaluated.

\section{Statistical analysis}

The data of the study were analyzed using the SPSS 22.0 for Windows (SPSS Inc., Chicago, IL, USA) computer program. Descriptive statistics were expressed as mean \pm standard deviation or median values and an inter quartile range (IQR) of 25-75\%, while categorical variables were expressed as numbers and percentage (\%). Kolmogorov-Smirnov test was used for normality distribution of the data. While the significance of the difference between the groups in terms of continuous numerical variables in which parametric test statistics assumptions were provided was examined with Student's t test, the significance of the difference in terms of continuous numerical variables where parametric test statistics assumptions were not met was evaluated with the MannWhitney U and Kruskal-Wallis tests. Chi-square and Fisher's exact test were used to analyze whether there was a relationship between categorical variables. The variables that may be effective for mortality were evaluated using the "enter" method in logistic regression analysis. The ROC curve was drawn to investigate the diagnostic value of the ferritin/lymphocyte percentage ratio. $p<$ 0.05 was considered statistically significant. Results were given at $95 \%$ confidence interval.

\section{Ethics statement}

Ethical approval and the necessary permissions were obtained from the ethics committee of our hospital (2011-Kaek-25 2020/0514), Ministry of Health, Directorate General of Health Services, and Directorate General of Public Health of Turkey. PCR test results of the patients were recorded and reported as part of the data of the Republic of Turkey, Ministry of Health, Public Health Management System, and Case Tracking Module.

\section{Results}

A total of 5,530 patient files were scanned retrospectively. While 732 patients were hospitalized for probable COVID-19, PCR test results of only 357 patients were found to be positive, out of whom 26 were excluded from the study due to various reasons. A total of 331 patients were included in the study.

The median age in the study population was 56 (IQR: 25 th75 th percentiles 46-66) while the median age values in critical and mild severity groups were 72.5 (IQR: 25 th-75th percentiles $64,75-79,25)$ and 52 ((IQR: 25th-75th percentiles 41-62), respectively. A proportion of $52.6 \%$ of the patients $(n=174)$ were female; $53.2 \%(\mathrm{n}=176)$ had comorbidities, namely hypertension $(20.2 \%, \mathrm{n}=67)$, diabetes mellitus $(5.4 \%, \mathrm{n}=18)$, chronic obstructive pulmonary disease (COPD) $(2.1 \%, \mathrm{n}=7)$ or multiple comorbidities $(19 \%, n=66)$. While $12.7 \%(n=42)$ of the patients had a clinically serious manifestation, $33.5 \%(n=111)$ had a typical CT finding according to the classification of the Radiological Society of North America Expert Consensus Statement on Reporting Chest CT Findings Related to COVID-19. The most common symptom in the patients was cough $(20.8 \%, \mathrm{n}=69)$. The 28 -day mortality was $12.4 \%(n=41)$ (Tab. 1$)$.

Demographic characteristics and clinical findings as per severity of disease category are listed in Table 2.

The median fever level of the patients was $37.0{ }^{\circ} \mathrm{C}$ (Min: 35.0, Max: 39.0), median saturation level was 97 (Min: 60, Max: 99), median pulse rate per minute was 100 (Min: 56, Max: 148), 
Tab. 4. Analysis of Variables with the Mann-Whitney U test.

\begin{tabular}{llrccc}
\hline & Mortality in 28 days & $\mathrm{n}$ & $\begin{array}{c}\text { Median } \\
\text { (IQR: 25th-75th percentiles) }\end{array}$ & $\mathrm{p}$ \\
\hline \multirow{3}{*}{ Lymphocyte } & Survival & 290 & $25.70(18.85-34.82)$ & \\
& Mortality & 41 & $10.10(6.65-16.40)$ & $<0.001$ \\
& Total & 331 & $24.60(14.00-33.60)$ & \\
\multirow{3}{*}{ Ferritin } & Survival & 290 & $167.15(68.72-308.37)$ & \\
& Mortality & 41 & $794.00(566.80-1264.00)$ & $<001$ \\
& Total & 331 & $188.00(79.06-416.10)$ & \\
FLPR & Survival & 290 & $6.55(2.47-13.07$ & $<0.001$ \\
& Mortality & 41 & $83.15(31.67-184.08)$ & \\
\hline
\end{tabular}

FLPR - Ferritin/ lymphocyte percentage ratio

Tab. 5. Kruskal Wallis-H test for FLRP and disease severity.

\begin{tabular}{llrcc}
\hline & Severity & $\mathrm{n}$ & $\begin{array}{c}\text { Median } \\
\text { (IQR: 25th-75th percentiles) }\end{array}$ & $\mathrm{p}$ \\
\hline \multirow{4}{*}{ FLPR } & Critical & 42 & $84.62(39.76-148.60)$ & \\
& Serious & 49 & $27.32(14.18-59.03)$ & $<0.05$ \\
& Moderate & 131 & $6.24(2.42-10.35)$ & \\
& Mild & 109 & $3.11(1.91-8.08)$ & \\
\hline
\end{tabular}

FLPR - ferritin/lymphocyte percentage ratio

Tab. 6. Investigation of the usefulness of the FLPR in the prediction of mortality.

\begin{tabular}{lccccc}
\hline AUC $(95 \%$ CI $)$ & $\mathrm{p}$ & Risk factor & Cut-off value & Sensitivity \% & Specificity \% \\
\hline & & & 9.8 & 97.6 & 65.2 \\
$0.909(0.857-0.961)$ & \multirow{2}{*}{0} & FLPR & 12.69 & 95.1 & 74.5 \\
& & & 15.59 & 90.2 & 78.3 \\
& & & 21.11 & 82.9 & 82.8 \\
\hline
\end{tabular}

AUC - Area Under Curve, FLPR - ferritin/lymphocyte percentage ratio

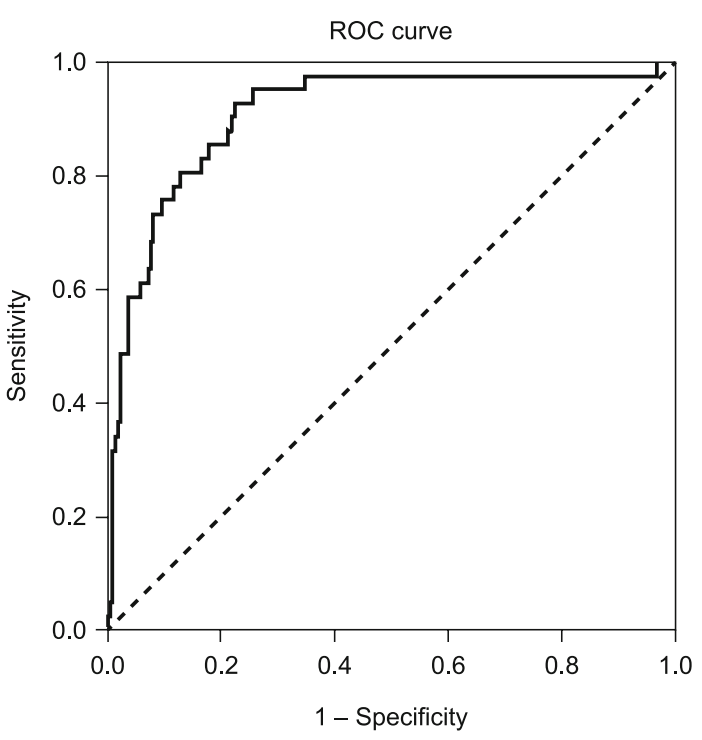

Fig. 1. ROC analysis for the ferritin/lymphocyte percentage ratio.

and median systolic blood pressure value was $110 \mathrm{mmHg}$ (Min: 70, Max: 180).

The measured mean lymphocyte percentage of the patients was $24.86 \pm 11.76$, the mean ferritin level was $346.39 \pm 443.88$ $\mathrm{ng} / \mathrm{mL}$ and the mean FLPR was $31.91 \pm 85.10$.
In the Chi-square test performed to analyze whether there was a relationship between CT findings and 28-day mortality, a statistically significant relationship was found $(\mathrm{p}<0.005)$. Additionally, a statistically significant relationship was found between the severity of the clinical status and 28-day mortality in the Chi-square test performed to analyze whether there was a relationship between the severity of the clinical status of the patients and the 28-day mortality $(p=0.000)$. Furthermore, there was a statistically significant relationship between the presence of comorbidities and the 28-day mortality in the Chi-square test performed to analyze whether there was a relationship between the presence of comorbidities and the 28-day mortality ( $\mathrm{p}<0.005$ ) (Tab. 3).

In the Kolmogorov-Smirnov test conducted to analyze the normality distribution it was observed that the data were not normally distributed. Therefore, Mann-Whitney $U$ test was performed to investigate whether there was a difference between mean FLPR levels, ferritin levels and lymphocyte percentage alone and 28-day mortality. As a result of this test, it was seen that FLPR levels were significantly higher in those who died in 28 days $(\mathrm{p}<0,01)$. When evaluated alone, ferritin levels were high and lymphocyte percentages were low in the mortality group $(p<0.001)$ (Tab. 4).

In the Kruskal Wallis-H test performed to determine whether the mean FLPR levels of the patients differ significantly according to the severity classification variable, the difference between the severity groups and the mean FLPR was found to be statistically significant $(\mathrm{x} 2=148,284 ; \mathrm{SD}=3 ; \mathrm{p}=0.000)$. It was observed that FLPR levels were high in groups with critical and serious clinical manifestations (Tab. 5).

In the logistic regression analysis performed to investigate whether the gender and comorbidities had an effect on 28-day mortality, it was seen that comorbidities were an independent risk factor.

The ROC curve presented in Figure 1 and the analyses presented in Table 6 were conducted to investigate the usefulness of FLPR in predicting mortality. In the ROC analysis for the FLPR level, the area under the curve (AUC) value was found to be 0.909 (95\% CI 0.857-0.961) (Fig. 1). When the cut-off value of FLPR was 9.80 , the sensitivity was found to be $97.6 \%$ and specificity was $65,2 \%$. When the cut off value of FLPR was 21.11 , the sensitivity was found to be $82.9 \%$ and specificity was $82.8 \%$ (Tab. 6).

\section{Discussion}

COVID-19 disease is a systemic infection commonly seen in males and individuals with comorbidities. Although its pathophysio- 
logy has not been fully elucidated, it manifests with hematological findings and increased levels of proinflammatory cytokines associated with pulmonary infection and severe lung damage $(3,15$, and 16). In our study, the presence of chronic disease was found to be an independent risk factor for 28-day mortality, which is consistent with the results found in literature.

The patients with critical/severe COVID-19 have a poor prognosis and high mortality rates. In studies, the rate of patients with critical/serious manifestation ranges between $18.9 \%$ and $63.3 \%$ (16-18). In our study, this rate was $27.5 \%$. The number of patients receiving intensive care and mechanical ventilation support is similar to that found in the literature.

In various studies, the mortality rate varies between $5 \%$ and $7.2 \%$ and this rate is correlated with age $(19,20)$. In our study, the patients were evaluated for 28-day mortality and the rate was found to be $12.4 \%$. According to the clinical classification, the mortality is relatively high in the critical and serious groups, and the coexistence of typical CT findings in these groups is remarkable.

COVID-19 is a systemic infection affecting the hematological system. Lymphopenia is used especially as a prognostic cardinal finding. In a study conducted by Guan et al. on 1,099 COVID-19 patients, the rate of lymphopenia was found to be $83.2 \%$ and it increased to $96.1 \%$, especially in critically ill patients (21). In addition to the use of lymphopenia as a poor prognosis marker, there are also studies showing that low lymphocyte values are associated with the development of ARDS (12).

The neutrophil/lymphocyte ratio (NLR) and lymphocyte percentage were frequently evaluated after determining the prognostic significance of lymphopenia. As NLR increased, the percentage of lymphocytes was found to be significantly lower in COVID-19 patients as compared to healthy volunteers, and this decrease was more obvious in the severely and critically ill patients. $(7,22$, 23). It is observed that the percentage of lymphocytes tends to decrease even if the lymphocyte count is within the normal range. The decrease in lymphocyte percentage is thought to be developing due to the destruction of erythrocytes infected with secondary hemophagocytic lymphohistiocytosis, and thus the ferritin level increases simultaneously after destruction $(5,18)$.

Lymphopenia and ferritin are used as separate prognostic markers, but the decrease in lymphocyte percentage and increase in ferritin level appear to be related to the mutual destruction of hematological cells caused by the SARS-COV2 virus. The complications that develop with the cytokine storm result in multiple organ failure and death (6). The FLPR may be the starting point of the aggressive immune response in the patient and may be prognostically significant.

The relationship of biochemical markers with the prognosis in COVID-19 was evaluated and the increase in serum ferritin level was associated with mortality and development of ARDS $(12,24)$. In the severely/critically ill patients, the mean ferritin level was $346.39 \pm 443.88 \mathrm{ng} / \mathrm{mL}$.

The combined use of hematological and biochemical parameters evaluated among poor prognostic markers may provide strong clues about the prognosis, as in the example of a decrease in the lymphocyte/c reactive protein ratio $(22,25)$. The FLPR evaluated for this purpose was found to be particularly high in the seriously and critically ill patient groups. The calculation of FLPR, especially as part of the first evaluation of patients in the emergency department, may be a guide in predicting the severity of the disease. Additionally, it can be used effectively when determining the need for clinical or intensive care hospitalization. Even if the clinical condition is good or stable, a high value of FLPR may give a clue about the prognosis of the disease and critically ill patients can be identified on time. When initiated early, the necessary treatment and interventions can be lifesaving.

\section{Conclusion}

In COVID-19 patients, it is important to use FLPR concurrently with patient clinical evaluations. When the cut-off value of FLPR is 9.80 , sensitivity is $97.6 \%$ and specificity is $65.2 \%$. When the cut-off value of FLPR is 21.11 , sensitivity is $82.9 \%$ and specificity is $82.8 \%$, which is effective in predicting mortality. Our study is the first study in the literature to use the FLPR. In this respect, it may contribute as an important mortality marker. We think that our study may form the basis for large-scale and comprehensive research.

\section{Limitations}

The most important limitation of our study is its retrospective character. Therefore, there was a loss during the collection of patient information and data.

The FLPR was calculated by recording the lymphocyte percentage values and ferritin level obtained with hemogram taken in the emergency room at the time of first admission. As we make the initial diagnosis and treatment in emergency department, we collect PCR tests and give first-line treatment, such that the admission day is the first day of diagnosis, but we do not categorize patients as to their symptomatic phase and day of symptoms.

\section{References}

1. Lu H, Stratton CV, Tang YW. Outbreak of pneumonia of unknown etiology in Wuhan, China: The mystery and the miracle. J Med Virol 2020; 92 (4): 401-402.

2. Hui DS, Azhar E, Madani TA et al. The continuing 2019-nCoV epidemic threat of novel coronaviruses to global Whealth - The latest 2019 novel coronavirus outbreak in Wuhan, China. Int J Infect Dis 2020; 91 : 264-266.

3. WHO. Coronavirus disease 2019 Situation Report 51 [Internet] 2020 Mar 11. Available from: https://www.who.int/docs/defaultsource/coronaviruse/situation-reports/20200311-sitrep-51-covid-19.pdf.

4. Xu H, Zhong L, Peng $\mathbf{J}$ et al. High expression of ACE2 receptor of 2019-nCoV on the epithelial cells of oral mucosa. Int J Oral Sci 2020; 12 (1): 8 .

5. Liao YC, Liang WG, Chen FW et al. IL-19 induces production of IL-6 and TNF-alpha and results in cell apoptosis through TNF-alpha. J Immunol 2002; 169 (8): 4288-4297. 


\section{9-804}

6. Aggarwal S, Gollapudi S, Gupta S. Increased TNF-alpha-induced apoptosis in lymphocytes from aged humans: changes in TNF-alpha receptor expression and activation of caspases. J Immunol 1999; 162 (4): 2154-2161.

7. Curbelo J, Bueno SL, Galvan-Roman JM et al. Inflammation biomarkers in blood as mortality predictors in community-acquired pneumonia admitted patients: Importance of comparison with neutrophil count percentage or neutrophil-lymphocyte ratio. PLoS One 2017; 12 (3): e0173947.

8. Reddan DN, Klassen PS, Szczech LA et al. White blood cells as a novel mortality predictor in haemodialysis patients. Nephrol Dial Transplant 2003; 18 (6): 1167-1173.

9. Kuwae N, Kopple JD, Kalantar-Zadeh K. A low lymphocyte percentage is a predictor of mortality and hospitalization in hemodialysis patients. Clin Nephrol 2005; 63 (1): 22-34.

10. Shoenfeld Y. Corona (COVID-19) time musings: Our involvement in COVID-19 pathogenesis, diagnosis, treatment and vaccine planning. Autoimmun Rev 2020; 19 (6): 102538.

11. Zhou F,Yu T, Du R et al. Clinical course and risk factors for mortality of adult inpatients with COVID-19 in Wuhan, China: a retrospective cohort study. Lancet.2020; 395 (10229): 1054-1062.

12. Wu C, Chen X, Cai Y et al. Risk Factors Associated With Acute Respiratory Distress Syndrome and Death in Patients With Coronavirus Disease 2019 Pneumonia in Wuhan, China. JAMA Intern Med 2020; 180 (7): 934-943.

13. Simpson S, Kay FU, Abbara S et al. Radiological Society of North America Expert Consensus Statement on Reporting Chest CT Findings Related to COVID-19. Endorsed by the Society of Thoracic Radiology, the American College of Radiology, and RSNA - Secondary Publication. J Thorac Imaging 2020; 35 (4): 219-227.

14. Verity R, Okell LC, Dorigatti I et al. Estimates of the severity of coronavirus disease 2019: a model-based analysis. Lancet Infect Dis 2020; 20 (6): 669-677.
15. Huang C, Wang Y, Li X et al. Clinical features of patients infected with 2019 novel coronavirus in Wuhan, China. Lancet.2020; 395 (10223): 497-506.

16. WHO. Report of the WHO-China joint mission on coronavirus disease 2019 (COVID-19) 2020, Geneva.

17. Li K,Wu J, Wu F et al. The Clinical and Chest CT Features Associated With Severe and Critical COVID-19 Pneumonia. Invest Radiol 2020; 55 (6): 327-331.

18. Qin C, Zhou L, Hu Z et al. Dysregulation of Immune Response in Patients With Coronavirus 2019 (COVID-19) in Wuhan, China. Clin Infect Dis 2020; 71 (15): 762-768.

19. Li LQ, Huang T, Wang YQ et al. COVID-19 patients' clinical characteristics, discharge rate, and fatality rate of meta-analysis. J Med Virol 2020; 92 (6): 577-583.

20. Onder G, Rezza G, Brusaferro S, Case-Fatality Rate and Characteristics of Patients Dying in Relation to COVID-19 in Italy. Jama 2020; 323 (18): $1775-1776$.

21. Guan WJ, Ni Z, Hu Y et al. Clinical Characteristics of Coronavirus Disease 2019 in China. N Engl J Med 2020; 382 (18): 1708-1720.

22. Lagunas-Rangel FA, Neutrophil-to-lymphocyte ratio and lymphocyteto-C-reactive protein ratio in patients with severe coronavirus disease 2019 (COVID-19): A meta-analysis. J Med Virol 2020; 92 (10): 1733-1734.

23. Yufei Y, Mingli L, Xuejao L et al. Utility of the neutrophil-to-lymphocyte ratio and C-reactive protein level for coronavirus disease 2019 (COVID-19). Scand J Clin Lab Invest 2020; 80 (7): 536-540

24. Terpos E, Ntanasis-Stathopulos I, Elalamy I et al. Hematological findings and complications of COVID-19. Am J Hematol 2020; 95 (7): 834-847.

25. Okugawa Y,Toiyama Y, Yamamoto A et al. Lymphocyte-C-reactive Protein Ratio as Promising New Marker for Predicting Surgical and Oncological Outcomes in Colorectal Cancer. Ann Surg.2020; 272 (2): 342-351

Received April 19, 2021. Accepted May 4, 2021. 